\title{
Search for multiple targets: Remember the targets, forget the search
}

\author{
TODD S. HOROWITZ and JEREMY M. WOLFE \\ Brigham \& Women's Hospital and Harvard Medical School, Boston, Massachusetts
}

\begin{abstract}
Models of visual search performance typically assume that search proceeds by sampling without replacement. This requires memory for each deployment of attention. We tested this assumption of memorydriven search using a multiple-targetsearch paradigm. We held total set size constant, varied the number of targets in the display, and asked subjects to report whether or not there were at least $n$ targets present, where $n$ was varied by block. This allowed us to measure the time to find each subsequent target. Memory-driven search predicts that reaction time should be a linear function of $n$. The alternative memory-free search hypothesis predicts an accelerating function. The data falsify the memory-driven hypothesis. They were consistent with the memory-free search hypothesis but would also be consistent with memory for a small number of previously attended locations.
\end{abstract}

Visual search is a common, everyday behavior that requires close cooperation between memory and attention. Memory is required for any search for a specific target. Prospective memory tells us what to look for and when. Target templates held in working memory help direct attention. When a likely target object is attended, its representation in short-term visual memory serves as a probe into long-term memory to retrieve its identity, which must then be matched against the template in working memory. Implicit memory for past searches can influence subsequent searches (Chun \& Jiang, 1998; Hillstrom, 2000; Maljkovic \& Nakayama, 1994, 1996).

Naturally then, memory plays a key role in major theoretical approaches to search and attention (e.g., Chun \& Potter, 1995; Duncan \& Humphreys, 1989, 1992; W. Schneider $\&$ Shiffrin, 1977). Some researchers have gone so far as to propose that working memory is attention (W. X. Schneider, 1995), and there are data to suggest that the contents of working memory help direct attention (Downing, 1999). The connection between visual search and memory search has been empirically demonstrated by Hillstrom and Logan (1998), who showed substantial training transfer between the two tasks.

This paper is concerned with an additional form of memory, widely held to contribute to the efficiency of visual search: memory for each deployment of attention during a search. Accounts of visual search performance

This research was supported by grants from AFOSR and by NIH Grants EY05087, MH1 1306-01, and HL 07609-13. The authors thank Laura Adams, George Alvarez, Peter Brawn, Serena Butcher, Paul Downing, Art Kramer, Gary Randall, Roger Remington, Steve Yantis, and an anonymous reviewer for helpful comments on the manuscript and Kari Dahlen and Nicole Klempen for collecting the data. Correspondence should be addressed to T. S. Horowitz, Center for Ophthalmic Research, Brigham \& Women's Hospital, 221 Longwood Ave., Boston, MA 02115 (e-mail: toddh@ search.bwh.harvard.edu). typically assume (implicitly or explicitly) that subjects search through the items in the display one by one, without retracing their steps,${ }^{1}$ or, in terms of probability theory, that visual search proceeds by sampling without replacement. In order for this to occur, there has to be some memory mechanism that keeps track of previously attended locations. This assumption of memory-driven search is a central tenet of the standard self-terminating serial processing model (Sternberg, 1969), which has been assumed in almost all models of visual search performance with a serial component for the last 30 years (e.g., Grossberg, Mingolla, \& Ross, 1994; W. Schneider \& Shiffrin, 1977; Treisman \& Gelade, 1980; Treisman \& Sato, 1990; Wolfe, 1994).

The empirical support for memory-driven search is surprisingly thin given its widespread acceptance in models of search. The leading hypothesis for this form of memory in search was proposed by Posner and Cohen (1984), who proposed that inhibition of return (IOR; the tendency not to return to recently attended locations in orienting experiments) served to prevent attention from being deployed to rejected distractors. Klein (1988) devised an elegant experiment to test this hypothesis. Subjects were asked to perform a visual search task, which could be either highly efficient ( $\mathrm{Q}$ among Os) or inefficient (O among Qs). After $25 \%$ of trials, subjects were required to make a speeded detection response to a luminance probe, which could appear either at a location previously occupied by a distractor ("on probes") or at a previously blank location ("off probes"). Reaction time (RT) was significantly slower for on probes than for off probes, providing support for the IOR version of the memory-driven assumption. Though this finding initially did not replicate (Klein \& T. L. Taylor, 1994; T. L. Taylor \& Klein, 1998; Wolfe \& Pokorny, 1990), recent work suggests that this effect can be observed providing the search stimuli persist into the probe phase (Müller \& von Mühlenen, 2000; Takeda \& Yagi, 2000). 
It is easy to see the advantages for the visual system in maintaining such a memory. If rejected distractors (or their locations) are inhibited, the system would avoid potentially costly duplication of effort and could locate and identify targets more efficiently. However, there may also be disadvantages to this scheme. In the dynamic natural world, as opposed to the static milieu of the typical laboratory search experiment, the contents of the scene may change rapidly or the initial identification of an object may be faulty. IOR would not be an advantage if a predator was initially and irrevocably misidentified as a rock. Furthermore, although subjects in laboratory experiments may often wish for a memory system that stores every pixel of every image, every saccade, and every deployment of attention, the brain tends to discard unimportant details, retaining only the level of detail that is practical (given a finite brain) and adaptive (given the structure of the environment). Memory-driven search should be treated as an empirical issue, rather than taken as given.

In a recent paper (Horowitz \& Wolfe, 1998), we claimed that visual search is actually "memory-free," by which we meant that no record was being kept of the deployments of attention during a search. Our experiments involved a "dynamic search" condition, in which we disrupted this hypothetical memory for deployments of attention during a trial by replotting all items at new, randomly chosen locations every $100 \mathrm{msec}$. If normal search through static stimuli is memory driven, performance should have been disrupted on dynamic trials. However, if search is normally memory free, then the efficiency of search should have been comparable for static and dynamic search. In several experiments, we found that RT $X$ set size slopes were equivalent for the two conditions, contradicting the memory-driven hypothesis (Horowitz $\&$ Wolfe, 1998). In the present paper, we present converging evidence for the memory-free search hypothesis from a simple variant of a standard, static search paradigm that does not involve actively disrupting processing.

Mathematically, the difference between memory-driven search and memory-free search is the difference between sampling without replacement and sampling with replacement. We can therefore think of visual search in terms of the classic probability theory problem of pulling colored balls from an urn (Johnson \& Kotz, 1977). Assume that distractors are red balls and targets are green balls. Sampling without replacement (memory-driven search) means that the subject pulls balls from the barrel, examines their color, and tosses them aside. The number of draws, $S$, from the barrel before one green ball is pulled out is given by the negative hypergeometrical distribution (Johnson \& Kotz, 1977). The expected value $E(S)$ of this distribution, assuming $t$ targets and $d$ distractors, is given by

$$
E(S)=\frac{(t+d+1)}{t+1} .
$$

For a memory-free model, search proceeds with replacement: The subject pulls a ball from the urn, classifies it, and then returns it to the urn before drawing the next ball. In this case, the distribution of finishing times is given by the negative binomial distribution (Johnson \& Kotz, 1977), and the expected value of $S$ is

$$
E(S)=1+\frac{d}{t} .
$$

In the majority of search experiments in the literature, only one target is present $(t=1)$. In this case, Equation 1 reduces to $E(S)=(d+2) / 2$, which leads to the assumption that only half of the items are sampled on average during a serial search. Equation 2 reduces to $E(S)=d+1$, indicating that, on average, as many samples as there are items (plus one) are needed to find the target during a memory-free serial search. The predictions of our previous, dynamic search experiments implicitly assume this difference in the expected number of samples (Horowitz $\&$ Wolfe, 1998). If we wish to test the memory-free hypothesis with static stimuli, we cannot use simple search for a single target because we lack firm knowledge of the attentional dwell time (Moore \& Wolfe, in press; Moray, 1969). Thus, using RT data, we cannot determine whether subjects take $X$ samples at a rate of $Y$ samples/sec or $2 X$ samples at a rate of $2 Y$ samples/sec. However, Equations 1 and 2 suggest that multiple-target search tasks will be more informative. Again, let's assume that distractors are red balls and targets are green balls. Under the memorydriven model, sampling occurs without replacement, which means that the subject pulls balls from the barrel, examines their color, and tosses them aside. After the first green ball is found, the subject tosses it aside with the rest and keeps plucking balls from the barrel. When the subject goes to search for the second target, however, there are now fewer balls overall; the effective set size has decreased. The number of remaining green balls decreases proportionately, and, thus, the time to find each successive green ball is a constant.

More precisely, the time to find $n$ by targets sampling without replacement is given by the general form of Equation 1, the expected value of the negative hypergeometrical distribution (Johnson \& Kotz, 1977), shown in

$$
E(S)=\frac{n(t+d+1)}{t+1} .
$$

It is clear that if we hold $t$ and $d$ constant and vary $n$, the RT will be a linear function of $n$.

Under the memory-free model, however, the subject throws each ball back into the barrel after examining it. Thus, the effective set size remains constant. The dominant factor is now the decreasing number of remaining targets, and the time to find each successive target now lengthens. The necessary number of samples is again given by the negative binomial distribution. We can generalize Equation 2 by assuming that each search for a target is an independent search (an instance of Equation 2) and that each target, once found, becomes a distractor, thus decreasing the number of targets by one and increasing the number of distractors by one with each search. We formalize this argument in Equation 4: 


$$
E(S)=\sum_{i=1}^{n}\left[1+\left(\frac{d-i+1}{t+i-1}\right)\right] .
$$

Note that this illustrates the limited sense in which we label search as memory free. In order to propose this test, we must assume that a subject will be able to remember that he/she found a target at a specific location even if he/she forgets the course of the search that led to that target (a point demonstrated by Gibson, Li, Skow, Brown, $\&$ Cooke, 2000). Without some memory, the subject would not know whether he/she had found two targets in a display or the same single target twice. However, whatever form this memory takes, it does not guide attention. That is, a subject may remember seeing a stimulus (even a stimulus at a particular spatial location), but that need not prevent him/her from revisiting that location during the search process.

Figure 1 shows the number of samples predicted by each model as a function of $n$, assuming 16 items in the display, 5 of which are targets. Both models show increasing RTs as a function of $n$, but the memory-free curve accelerates, whereas the memory-driven curve is linear, reflecting the superior efficiency of the memory-driven model in this situation.

Our experiments were designed to test whether the empirical RT function for such a task would be linear or accelerating. We held total set size constant, varied the number of targets in the display, and asked subjects to report whether or not there were at least $n$ targets present. The criterion, $n$, was varied by block. There have been many studies on the effect of redundant targets in search displays (Estes \& H. A. Taylor, 1966; Ward \& McClelland, 1989; Wolford, Wessel, \& Estes, 1968), but these have typically been concerned with the effects of redundancy on RT to the first target. In contrast, we were inter-

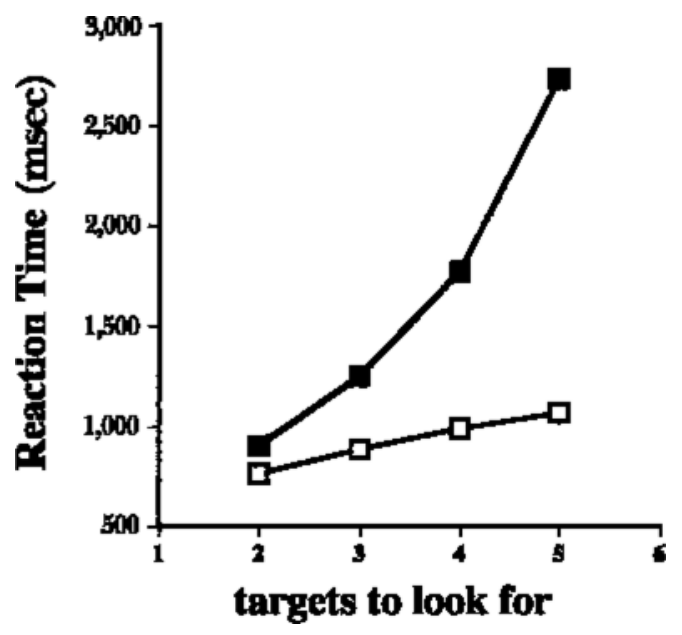

Figure 1. Simulated reaction times (assuming a 50-msec dwell time) to find a given number of targets, assuming a display consisting of 5 targets and 11 distractors, for memory-driven (Equation 3 ; open symbols) and memory-free (Equation 4; filled symbols) models. ested in measuring RT to each subsequent target, which this paradigm allowed us to do.

\section{METHOD}

\section{Subjects}

Ten subjects from our volunteer subject pool served as subjects in return for compensation of $\$ 7 /$ hour. All subjects had normal or corrected-to-normal visual acuity and passed the Ishihara color screen. All gave informed consent prior to their participation.

\section{Apparatus and Stimuli}

Set size was fixed at 16 items, presented at randomly chosen locations in a $6 \times 6$ matrix. Each cell was $1.17^{\circ}$ from its neighbor. Distractors were letters chosen from the set of all letters, except for I, J, and $\mathrm{O}$, and were presented in 48-point Arial font. In a given display, each distractor was unique. Two versions of the experiment were run. In the varied-targets condition, targets were numerals, chosen randomly (without replacement) on each trial from the set of nonzero numerals (zero was excluded to avoid confusion with the letter $\mathrm{O}$ ). In the identical-targets condition, all targets on a given trial were identical to each other, again chosen randomly from the digits 1 through 9.

\section{Procedure}

Each session consisted of four blocks of 240 trials. The identicaltargets and varied-targets conditions were run in separate 1-h sessions, with condition order counterbalanced. At the start of each block, the subject was given the following instructions:

\begin{abstract}
In this block, we want you to tell us whether or not there are $n$ numerals in the display. There may be anywhere from 1 to 5 numerals present. If you see at least $n$ numerals, press the "quote" key, but if you only see $\mathrm{n}-1$ or fewer numerals, press the "a" key. Please respond as soon as you're sure how many numerals there are in the display, because we are interested in your reaction time. There will be 30 practice trials and 240 real trials.
\end{abstract}

The number $n$ was constant over a block but different for each block, and the order of blocks was randomly selected for each subject. In each block, half of the trials required a "yes" response, and half required a "no" response. This meant, for instance, that when $n$ was 5 , half of the trials (120) had 5 targets, whereas there were 30 trials each with $1,2,3$, and 4 targets. The subjects were asked to respond as quickly and as accurately as possible.

\section{RESULTS}

Figure 2 shows correct target-present RTs, averaged across subjects for the two versions of the experiment. Error bars are standard errors of the mean. Qualitatively, the answer is quite clear. The curves appear to be accelerating, as predicted by the memory-free hypothesis, and not linear, as predicted by the memory-driven hypothesis.

\section{Statistical Analysis}

To provide a statistical test for this impression, we concentrate on the functions for 4 and 5 targets present since the functions for 2 and 3 targets present lack enough points to evaluate the linearity of the curve. Our analysis focused on identifying a significant quadratic trend and then determining the sign of the quadratic regression coefficient. A positive quadratic regression coefficient would indicate a positively accelerated curve. Results of these analyses are given in Table 1 . 

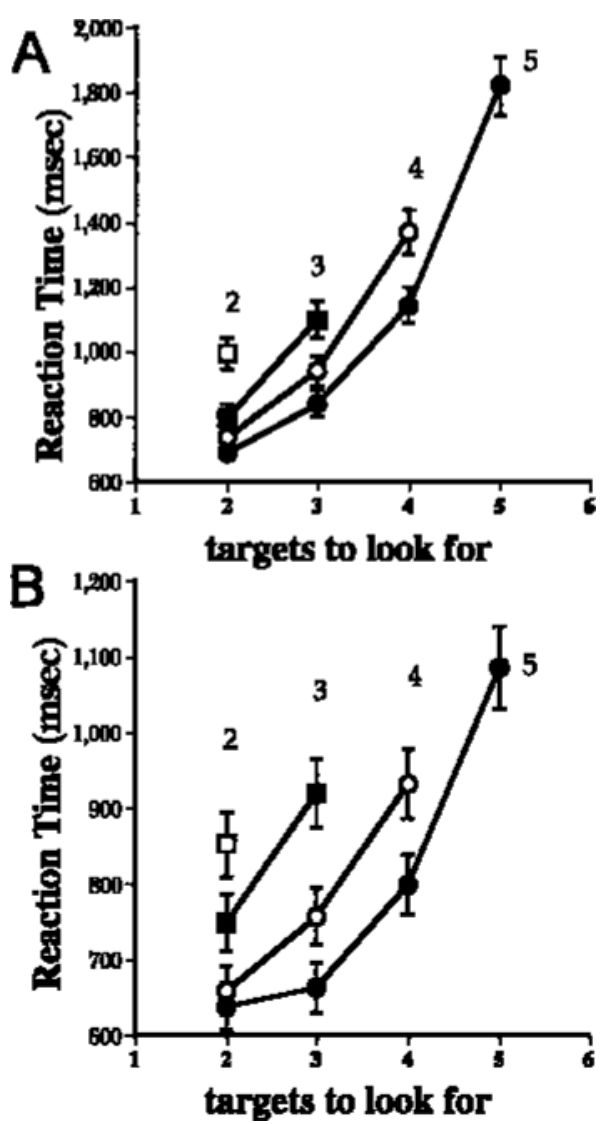

Figure 2. Correct "yes" reaction times. Panel A shows results from the varied-targets condition; panel $B$ shows data from the identical-targets condition. Parameters on the curves refer to the number of targets actually present $(m)$. Error bars indicate the standard error of the mean. Note the different scales on the two panels.

These analyses confirm what is obvious from a glance at Figure 2: The $n \times$ RT curves are positively accelerated. The effect is hard to confirm with only three points on a curve (the 4-target case) but becomes statistically robust when there are four points to evaluate (the 5-target case). This pattern of results makes little sense if one assumes a memory-driven search mechanism; a search that proceeded through the display should find each successive target in the same amount of time. To explain these data on a memory-driven account, we would have to assume that the inhibitory memory was reset after each target was found, which would seem to limit the usefulness of the proposed mechanism for any but the simplest of search tasks.

Another striking feature of the data is that the variedtargets condition produced slower RTs than did the identical-targets condition. We confirmed this observation via a series of separate analyses of variance (ANOVAs) performed on the data at each level of $m$, the number of targets actually present. The main effects ${ }^{2}$ of varied versus identical targets are shown in Table 2. Correct "no" RTs are shown in Figure 3. Unlike the curves for "yes" RTs, the functions in Figure 3 are linear and flat. According to the standard self-terminating serial search model, an exhaustive search should always be required to determine that there are fewer than $n$ targets. ${ }^{3}$ Since total set size was held constant in this experiment, the "no" trial RT curves should lie on top of one another. In fact, each additional target in the display increased RTs in the variedtargets condition by around $500 \mathrm{msec}$. We statistically confirmed this observation by submitting the data from each block of trials (where $n$ is held constant) to a one-way ANOVA and testing the effect of $m$. Results are given in Table 3.

According to the memory-free model, subjects give up searching and respond "no," not when they have searched all the items but when they feel that they have searched "long enough." The criterion for what is "long enough" will vary with many factors (Chun \& Wolfe, 1996). We can speculate as to why subjects are willing to wait longer when there are more targets in the display, but the critical point is that subjects are not engaging in exhaustive search but are adjusting some criterion that varies directly with the number of targets (Zenger \& Fahle, 1997).

Subjects can commit two types of errors in this task: underestimates (i.e., responding "no" when the correct answer is "yes"; shown in Figure 4) and overestimates (i.e., responding "yes" when the correct answer is "no"; shown in Figure 5). We use the terms underestimates and overestimates, rather than the traditional misses and false alarms, because errors in this task are not directly comparable to misses and false alarms in standard search tasks. In a standard present/absent search, a "no" response on a targetpresent trial indicates that the target has been missed. However, if a subject is asked whether or not there are 5 targets in a 5-target display, and he/she responds "no," he/she might have missed 1 target or 2 , or 5 . Perhaps, the subject found all 5 and just miscounted. It is simply not possible to determine the actual number of misses in this task.

Looking at Figures 4 and 5, it would appear that the two types of errors behave differently, with underestimatescovarying with correct "yes" RTs and overestimates moving in the opposite direction. In fact, the error data follow a fairly simple pattern: The closer the number of targets in the display $(m)$ is to the number of targets being looked for $(n)$, the more errors subjects make. This is similar to the "distance effect" observed for numerical comparisons in RT (Moyer \& Landauer, 1967). The presence of a distance effect is not surprising in this experiment. When $m$ is close to $n$, more distractor items have to be searched. That is, determining whether there are at least 2 targets present can be done more quickly when there are actually 5 targets, as opposed to when there are actually 2 targets. This, in turn, will increase the chance of producing an error, because each time an item is attended, there is some probability of it being misidentified. Moreover, when $m$ is close to $n$, there is a greater chance of a decision stage 
Table 1

Statistical Analyses of the Effect of $n$ on "Yes"

Trial Reaction Times for the $m=4$ and $m=5$ Curves

\begin{tabular}{|c|c|c|c|c|c|c|c|c|c|c|}
\hline \multirow[b]{2}{*}{$\underline{n}$} & \multicolumn{3}{|c|}{ Main Effect } & \multicolumn{3}{|c|}{ Quadratic Trend } & \multicolumn{4}{|c|}{ Quadratic Regression } \\
\hline & $F$ & $d f$ & $p$ & $F$ & $d f$ & $p$ & Mean Coefficient & $t$ & $d f$ & $p$ \\
\hline \multicolumn{11}{|c|}{ Varied Targets } \\
\hline 4 & 29.42 & $(2,18)$ & $<.0001$ & 2.357 & $(1,9)$ & $>.1$ & 91.63 & 2.821 & (9) & $<.05$ \\
\hline 5 & 61.39 & $(3,27)$ & $<.0001$ & 16.72 & $(1,9)$ & $<.0005$ & 261.67 & 7.11 & (9) & $<.0001$ \\
\hline \multicolumn{11}{|c|}{ Identical Targets } \\
\hline 4 & 13.60 & $(2,18)$ & $<.0005$ & $<1$ & $(1,9)$ & $>.1$ & 32.42 & 1.64 & (9) & $>.1$ \\
\hline 5 & 28.25 & $(3,27)$ & $<.0001$ & 11.49 & $(1,9)$ & $<.005$ & 131.44 & 4.68 & (9) & $<.005$ \\
\hline
\end{tabular}

error. That is, it is easier to mistake 5 targets for 4 than it is to mistake 1 target for 4 .

Presumably, the subjects could have improved accuracy by paying a cost in slower RTs. How would that have changed the pattern of results shown in Figure 2? In the case of underestimate errors, the covariance with RT suggests that reducing errors would have made the acceleration of the functions in Figure 2 more pronounced. The only significant numbers of overestimation errors occur in the cases in which the number of targets to look for is one greater than the number of targets present. If the subjects had been more cautious and more accurate about these trials, the main effect would have been to increase RTs for "no" responses in those conditions. It is possible that some of these errors represent trials in which the subjects guessed quickly before they had enough information to make a correct response. On such trials, wrong guesses will show up as errors, but correct ("lucky") guesses will reduce the estimate of mean RT on "yes" trials. The "true" RTs should thus be longer (Eriksen, 1988; Miller \& Lopes, 1991). This would, again, increase the acceleration of the curves in Figure 2. To summarize the point, the subjects made a substantial number of errors, but the pattern of errors suggests that we may have merely underestimated the acceleration of the functions in Figure 2.

\section{Model Fitting}

We can do more than simply discriminate between two hypothetical curve shapes. In a standard search task, we can fit a linear model to the data and estimate parameters such as the intercept and the slope, which indexes search efficiency. Similarly, we can fit simple models to the data from the $n$-targets task, on the basis of either memorydriven or memory-free assumptions. We model RT by adding two parameters to Equations 3 and 4: an intercept, $b$, and a search rate, $r$. The search rate is equivalent to the slope of the linear regression on RT $\times$ set size data. The memory-driven model becomes

$$
\mathrm{RT}=b+\frac{n r\left(t+\frac{d+1)}{t}+1\right.}{1}
$$

and the memory-free model becomes

$$
\mathrm{RT}=b+\sum_{i=1}^{n}\left[1+\left(\frac{t-i+1}{d+i-1}\right)\right] r .
$$

Using these equations, we can solve for $b$ and $r$ and determine whether the estimates for $r$ agree with those derived from other search tasks, as well as quantitatively compare the goodness of fit of the two models.

In addition to the two "pure" models, we fit two hybrid models, a mixture model and a limited-capacity memory model, which will be discussed separately.

The mixture model. The mixture model assumes that subjects perform memory-guided searches on some trials and memory-free searches on the remaining trials. ${ }^{4}$ The observed distribution of RTs would then be a mixture of the two pure (memory-driven and memory-free) distributions, usually termed the basis distributions. Unfortunately, tests for the presence of mixture distributions typically require a priori knowledge of the basis distributions (Yantis, Meyer, \& Smith, 1991), which we do not have. Instead, we estimated how well a simple hypothetical mixture distribution could account for the mean RT data. The mixture model was implemented by assuming that each data point consisted of some proportion $(w)$ of memorydriven searches and some proportion $(1-w)$ of memoryfree searches. If the output of the memory-driven model in Equation 5 is set to $D$ and the output of the memoryfree model in Equation 6 is set to $F$, then the mixture model is given in Equation 7. The two component equations were constrained to use the same search rate and intercept parameters during model fitting.

$$
\mathrm{RT}=w D+(1-w) F .
$$

Each model was fit separately for each subject, using a least mean squared error criterion. All 10 hit data points from each subject were used to fit the models. The mean parameter estimates for the two models are compared in Table 4, along with the corresponding root mean square (RMS) errors.

Table 2

Main Effect of Identical Versus Varied Targets on Correct "Yes" Reaction Times for Each Value of $\boldsymbol{m}$, the Number of Targets Actually Present

\begin{tabular}{ccc}
\hline$m$ & $F(1,9)$ & $p$ \\
\hline 2 & 21.03 & $<.005$ \\
3 & 10.77 & $<.005$ \\
4 & 23.04 & $<.005$ \\
5 & 27.06 & $<.001$ \\
\hline
\end{tabular}



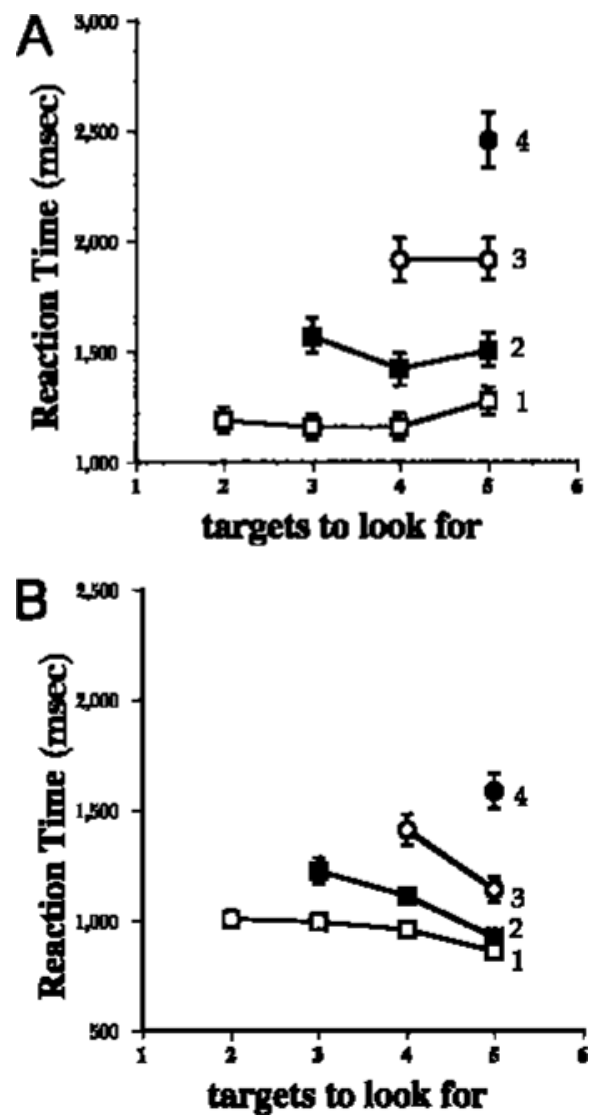

Figure 3. Correct reaction times from "no" response trials. Panel A shows results from the varied-targets condition; Panel B shows data from the identical-targets condition. The parameters indicate the number of targets actually present $(m)$. Note that the $m=1$ curve exists only for "no" trials, whereas the $m=5$ curve exists only for "yes" trials. Error bars indicate the standard error of the mean. Here, the two panels are plotted on the same scale.

The quantitative advantage of the memory-free model over the memory-driven model was not dramatic, but it was consistent. For the varied-targets case, the advantage of the memory-free model was significant $[t(9)=6.11$, $p<.0005]$, whereas, for the identical-targets data, the difference did not reach significance $[t(9)=1.71, p=.12]$. Although the mixed model improved the fit to the data when compared with the pure amnesic model, this improvement was not significant for either the varied-targets data $[t(9)=1.12, p=.29]$ or the identical-targets data $[t(9)=1.78, p=.10]$. Since this negligible improvement in the fit comes at the expense of an extra parameter $(w)$, the pure memory-free model remains a better description of the data.

The value of $w$ indicates that the subjects, on average, behaved as if they could use a memory-driven strategy on $11 \%$ of trials. In fact, for most subjects ( 7 of 10 in the varied-targets condition and 5 of 10 in the identical-targets condition), the best-fitting value for $w$ was 0 , indicating that the pure amnesic model fit better than any mixture. The highest observed weight in the varied-targets condi- tion was .66 (note that for this subject, the best-fitting intercept was an impossible $0 \mathrm{msec}$; the weight drops to .47 as the intercept is forced above $150 \mathrm{msec}$ ). For the identical-targets data, 1 subject had a weight of 1.0, indicating that the pure memory model fit better than any mixture.

The limited-capacity memory model. The pure memory-free model assumes no memory for prior attentional deployments whatsoever, whereas the memorydriven model assumes a perfect memory for rejected distractor locations without any capacity limits (or at least with a capacity of 16). The limited-capacity memory model, however, allows capacity to vary between 0 and infinity. A model with a limited capacity proposes that we can avoid retracing the last $C$ steps, where $C$ is a measure of the capacity. Such a memory might resolve some problems that a pure memory-free account creates. For instance, it is hard to imagine how to search with a memoryfree system without perseverating on the most salient item in the display, even if it were a distractor (Barbur, Forsyth, \& Wooding, 1993). With some memory for the most recently visited items, this problem is ameliorated. In order to produce the accelerating curves we see in this experiment, as well as to account for the apparently memoryfree behavior of subjects in our previous experiments, the memory capacity would have to be quite low. However, it need not be zero.

According to the memory-driven model, the next item to be examined is sampled from only all those items that have not been previously inspected. In the memory-free model, the next item is sampled from the full set of items, regardless of whether or not they have been previously examined. The limited-capacity memory model proposes that the next item is sampled from all items, except for the $C$ most recently examined items. When a new item is examined, it (or its location) is somehow marked. However, since there are only a limited number of markers, this means that a marker has to be removed from some other item, presumably the item that has been marked the longest. To borrow an analogy from Pylyshyn (1989), the guidance system has $C$ "fingers." It can put a finger on each item after it has been examined, to prevent it from being resampled. However, once the $C+1^{\text {th }}$ item is selected, finger 1 must be removed from item 1 and placed on item $C+1$; at this point, item 1 may again be sampled by the system, assuming that the target has not yet been found.

If all distractors are treated identically (and identified targets treated as distractors), this is mathematically identical to a model in which, once the fingers are down, they do not move; this version is more mathematically tractable.

Table 3

Statistical Analysis of the Effect of $m$ on "No" Trial Reaction Times

\begin{tabular}{ccccccc}
\hline & & \multicolumn{2}{c}{ Varied Targets } & & \multicolumn{2}{c}{ Identical Targets } \\
\cline { 3 - 4 } \cline { 6 - 7 }$n$ & $d f$ & $F$ & $p$ & & $F$ & $p$ \\
\hline 3 & $(1,9)$ & 40.68 & $<.001$ & & 76.27 & $<.001$ \\
4 & $(2,18)$ & 52.66 & $<.001$ & 63.32 & $<.001$ \\
5 & $(3,27)$ & 62.47 & $<.001$ & 66.59 & $<.001$ \\
\hline
\end{tabular}



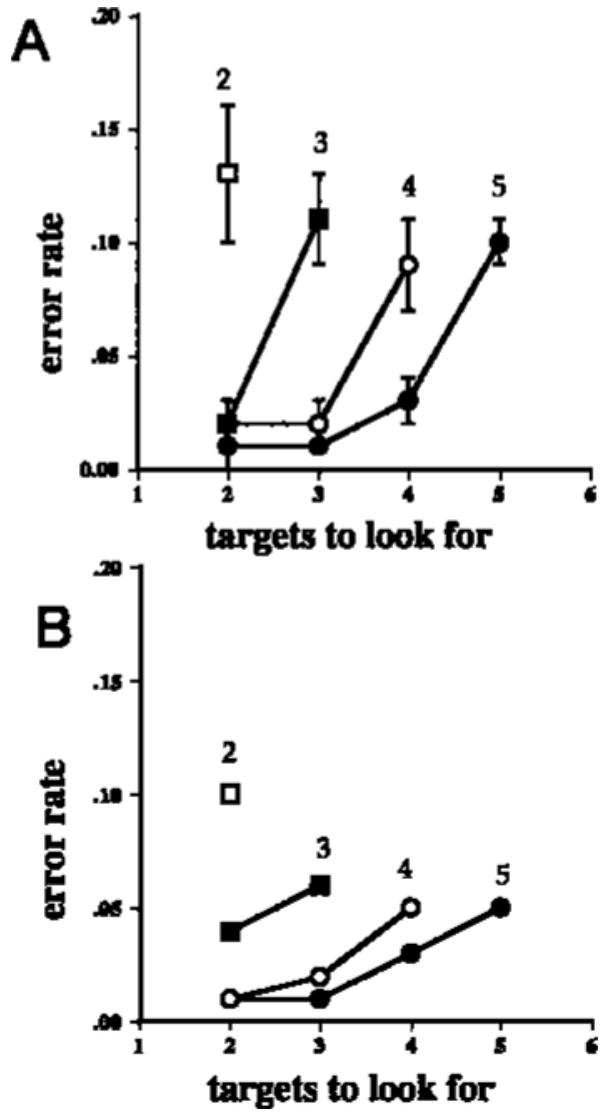

Figure 4. Proportion of underestimates (incorrectly responding “no"). Panel A shows results from the varied-targets condition; Panel B shows data from the identical-targets condition.Parameters on the curves refer to the number of targets actually present $(m)$. Error bars indicate the standard error of the mean.

If we label the capacity ("fingers," or the number of previous deployments that are remembered) as $C$, then the limited-capacity memory model assumes that each search consists of two phases: a memory-driven search through $C$ items, followed by a memory-free search through set size $-C$ items. Given $m$, the number of targets in the array, there will be $m^{\prime}$ targets in the initial set of $C$ items, and $m-m^{\prime}$ targets among the remaining items. The $m^{\prime}$ can take on values from 0 to $m$, or from 0 to $C$ if $C<m$. We calculated the probability of each possible outcome (i.e., $m^{\prime}=1$, and $m-m^{\prime}=4$ ). Next, for each outcome, we determined the time required to search through the initial $C$ items in memory-driven fashion and added it to the time needed to search through the remaining items in memory-free fashion (if $m^{\prime} \geq n$, the memory-free search was skipped; if $m^{\prime}<n$, search through the initial $C$ items was exhaustive), and we multiplied this time by the probability of that particular outcome. The weighted RTs were then summed to determine the predicted RT for each target-present data point in our experiment. As in the other models, these numbers were multiplied by a search rate, $r$, and added to an inter- cept, $b$. Then $r$ was constrained to be positive, $b$ was constrained to be $>150 \mathrm{msec}$, and $C$ was allowed to vary between 0 and $10 .{ }^{5}$ Results are shown in Table 5.

The first and most important point to note is that, as with the mixture model, the limited-capacity model does not provide a significant improvement over the pure memoryfree model, despite the luxury of an added parameter, $C$.

That said, it is possible to derive the memory capacity, $C$, that provides the best fit of each subject. The mean fitted value of the capacity of the memory estimate was 3 items. However, there is substantial variability in the estimates for different subjects. For 4 of 10 subjects, the best-fitting capacity was 0 , indicating a pure memoryfree model. For the remaining subjects, however, $C$ ranged as high as 9 items, which is fairly large. The best fit in the average data is for $C=0$; however, as $C$ goes from 0 to 10 , the RMS error increases by only $2.78 \mathrm{msec}$. So, although the pure memory-free model provides the most economical fit to the data, the predictions of this model (in this context) differ very little from the predictions of models that assume a limited memory for prior deployments of attention.
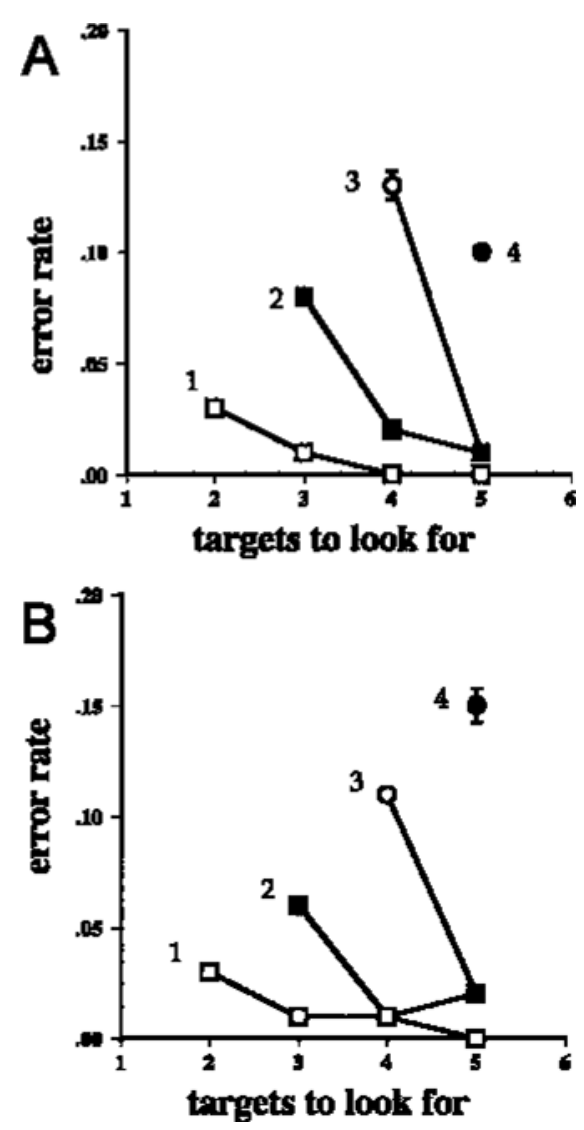

Figure 5. Proportion of overestimates (incorrectly responding "yes"). Panel A shows results from the varied-targets condition; Panel B shows data from the identical-targets condition. Parameters on the curves refer to the number of targets actually present $(m)$. Error bars indicate the standard error of the mean. 
Table 4

Model Fitting Results

\begin{tabular}{|c|c|c|c|c|c|c|}
\hline \multirow[b]{2}{*}{ Parameter } & \multicolumn{3}{|c|}{ Varied-Targets Condition } & \multicolumn{3}{|c|}{ Identical-Targets Condition } \\
\hline & $\begin{array}{c}\text { Memory-Free } \\
\text { Model }\end{array}$ & $\begin{array}{c}\text { Mixture } \\
\text { Model }\end{array}$ & $\begin{array}{c}\text { Memory-Driven } \\
\text { Model }\end{array}$ & $\begin{array}{c}\text { Memory-Free } \\
\text { Model }\end{array}$ & $\begin{array}{c}\text { Mixture } \\
\text { Model }\end{array}$ & $\begin{array}{c}\text { Memory-Driven } \\
\text { Model }\end{array}$ \\
\hline Search rate $(r)$ (msec/item) & 30.69 & 36.83 & 92.54 & 13.70 & 21.11 & 43.76 \\
\hline Intercept $(b)$ (msec) & 417.64 & 378.00 & 103.32 & 526.09 & 477.94 & 342.92 \\
\hline Weight $(w)$ & & 0.11 & & & 0.31 & \\
\hline RMS error (msec) & 159.50 & 159.15 & 177.36 & 76.55 & 75.87 & 94.24 \\
\hline
\end{tabular}

\section{DISCUSSION}

The requirements of this search task illustrate the interactions of attention and memory in visual search. Subjects must keep the task instructions and the current number of targets $(n)$ to find in working memory, retrieve visual descriptions of the nine digits from long-term memory, and use these descriptions to form target templates (Duncan \& Humphreys, 1992), which in turn must be held in working memory during each search trial. After finding each target, subjects must remember how many targets have already been found. Critically, however, subjects apparently do not remember which distractors they have rejected. The memory-free model fits the data better, both qualitatively and quantitatively. For the varied-targets condition, for all 10 subjects, the memoryfree model was a better fit than the memory-driven model. The parameters derived from the model fits are interesting (see Table 4). The memory-driven model yields a search rate of $93 \mathrm{msec} / \mathrm{item}$. This is much slower than previous data on search for numerals in letters (Hock, Rosenthal, \& Stenquist, 1985; Krueger, 1984) would suggest. The memory-free model, on the other hand, gives an estimate of $31 \mathrm{msec} /$ item, which is within the usual range for this type of search. Of course, although subjects may not keep track of identified distractors, they must keep track of identified targets in order to be able to do the task at all (Gibson et al., 2000). If attention is sampling the display with replacement, then it is possible to come upon the same target more than once during the course of the search. In order to perform with any reasonable level of accuracy, subjects must be able to remember whether or not they have already encountered a particular digit. What kind of memory subserves this function? In the varied-targets condition, the subjects could have maintained a verbal list of digits in working memory or a set of target locations in spatial memory. However, the results of the identical-targets condition indicate that it is possible to perform the task well without recourse to verbal memory, which would have been useless in this condition. ${ }^{6}$ The subjects in this condition must have been using some spatial memory to record where targets had been located. This representation clearly cannot be used to direct the deployment of attention, or else the subjects would surely have used it to remember distractor locations, and we would have observed linear functions.
The quadratic trend was less evident in the identicaltargets version, and the contrast between models correspondingly weaker, though still in favor of the memoryfree model, which produces better fits for 7 of the 10 subjects. This probably results from different search templates. In the varied-targets version of the experiment, since the targets are not known in advance, decisions cannot be made on the physical features of the stimuli, and all characters must be processed to the level of identity. In the identical-targets version, once the first digit is found, the subject knows the identity of the remaining targets and can adjust his/her search template accordingly. Depending on your preferred search model, this could result in suppression of features shared by letters and other digits, lowered decision thresholds, decreased targetnontarget similarity, and so on. Models aside, search becomes easier once you know that the targets are all " 3 "s, for example. This would explain the substantially faster responses we see in the identical-targets condition (Figure 2B). This identical-target benefit would tend to make search for each successive target more efficient. This would counter the increasing inefficiency of memory-free search, leading to the weaker advantage for the memoryfree model for this condition. Despite these likely effects, the memory-free model still provides a better characterization of the data in the identical-targets condition than does the memory-driven model.

\section{Inhibition of Return}

The idea that IOR functions as an inhibitory tagging system can be traced to Posner and Cohen (1984), who suggested that the function of IOR is to bias the organism toward novelty. IOR is typically observed in orienting experiments (Posner, 1980), in which subjects are asked to make a speeded response to the appearance of a target that appears in one of a limited number of locations. A cue (such as a luminance transient at the location) is used to

Table 5

Limited-Capacity Model Fitting Results

\begin{tabular}{lcc}
\hline & \multicolumn{2}{c}{ Condition } \\
\cline { 2 - 3 } \multicolumn{1}{c}{ Parameter } & Varied Targets & Identical Targets \\
\hline Search rate $(r)(\mathrm{msec} /$ item $)$ & 41.98 & 22.53 \\
Intercept $(b)(\mathrm{msec})$ & 372.17 & 480.72 \\
Capacity $(C)$ (items) & 3.00 & 5.00 \\
RMS error & 159.38 & 76.49 \\
\hline
\end{tabular}


direct attention to one of the possible locations, resulting in improved target detection at the attended locus relative to unattended loci, at least when the time between the onset of the cue and the onset of the target (stimulus onset asynchrony, or SOA) was brief. What Posner and Cohen observed, and other researchers have repeatedly confirmed (Maylor, 1985; Mondor, Breau, \& Milliken, 1998; Pratt, Kingstone, \& Khoe, 1997; Rafal, Calabresi, Brennan, \& Sciolto, 1989), was that this initial facilitation was replaced at longer SOAs by a long-lasting inhibitory effect: Targets are actually more difficult to detect at recently attended locations. The phrase inhibition of return reflects the theoretical assumption that this inhibitory effect is a result of the difficulty of redeploying attention to such locations. Posner and Cohen proposed that this tendency, while yielding less than ideal performance for laboratory subjects, had an adaptive value for an organism in the real world: IOR would prevent the organism from perseverating on one location or object in the visual field, thus encouraging exploration of the environment.

The explicit link between IOR and visual search was made by Klein (1988), building on earlier work by Briand and Klein (1987), Prinzmetal, Presti, and Posner (1986), and Treisman (1985). As described in the introduction, Klein found that, in conjunction search tasks (which should require attentional search; Treisman \& Gelade, 1980), luminance probes were harder to detect at distractor locations than at blank locations, whereas there was no effect for feature searches (which presumably do not require attentive search). As Klein and T. L. Taylor (1994) reported, the difference between the two search tasks in Klein (1988) appears to have been a statistical fluke. A number of replication attempts have shown slowed RTs to on probes relative to off probes in following either type of search task, and this slowing is attributed to forward masking (Klein, unpublished data described in Klein \& T. L. Taylor, 1994; Wolfe \& Pokorny, 1990). However, recent studies (Müller \& von Mühlenen, 2000; Takeda \& Yagi, 2000) have shown that the pattern of results initially reported by Klein can be observed if the search items remain visible during the probe stage, rather than being extinguished, as in the original experiments (and replications). This suggests an inhibition that is attached to object representations, rather than to spatial locations (Abrams \& Dobkin, 1994; Tipper, Driver, \& Weaver, 1991; Tipper, Weaver, Jerreat, \& Burak, 1994). It is not clear whether this phenomenon is more closely related to IOR or to visual marking (see below).

In the past decade, the boundary conditions for IOR have been explored and debated at length (Abrams \& Dobkin, 1994; Klein \& MacInnes, 1999; Klein \& T. L. Taylor, 1994; Reuter-Lorenz, Jha, \& Rosenquist, 1996; Snyder \& Kingstone, 2000; Tipper et al., 1991). The description of IOR that is emerging suggests that it might not be well suited to serving as an inhibitory tagging system for visual search, at least not at the level of modulating the rapid deployment of attention.
First, there are the well-known temporal dynamics of IOR. IOR takes $300 \mathrm{msec}$ to overcome the initial facilitation at an attended location (Posner \& Cohen, 1984). If attention is redeployed to a new stimulus every 25,50 , or even $100 \mathrm{msec}$, then IOR will not have time to influence the selection of the next item, though it could play a role in deployments of the eyes or of attention in long searches with large set sizes.

Second, there is widespread debate about whether IOR occurs during discrimination tasks, as opposed to simple detection tasks. Pontrefact and Klein (reported in Klein \& T. L. Taylor, 1994) and Terry, Valdes, and Neill (1994), among others, found no IOR in tasks in which subjects had to discriminate between targets, rather than simply making a speeded detection response, whereas Pratt and his colleagues (Pratt, 1995; Pratt \& Abrams, 1999; Pratt et al., 1997) have reported IOR in such tasks (see also Cheal, Chastain, \& Lyon, 1998; Oonk \& Abrams, 1998). If IOR is restricted to detection tasks, then we certainly should not expect a search task (which requires successive discriminations) to elicit IOR. Meanwhile, Lupiáñez (Lupiáñez, Milán, Tornay, Madrid, \& Tudela, 1997; Lupiáñez \& Milliken, 1999) has argued that the discrepancies between studies that find IOR for discrimination tasks and those that do not arise out of the different time courses of IOR following detection and discrimination. In Lupiáñez et al.'s experiments, IOR was first observed for detection tasks at a 400-msec SOA, but discrimination tasks did not show IOR until the 700-msec SOA. This brings us back to the time course issue: Such a mechanism is clearly not compatible with an attentional dwell time on the order of $50 \mathrm{msec}$ and thus cannot play much of a role in FIT-style theories of search. It is consistent with the 300- to 500-msec dwell time estimate given by attentional blink experiments (Chun \& Potter, 1995; Duncan, Ward, \& Shapiro, 1994). Such results support a limited-capacity parallel explanation for the processing of search displays, and such theories generally do not require an inhibitory tagging mechanism.

Finally, there may be a limit on the number of locations that can be concurrently inhibited. The vast majority of IOR experiments have been carried out with a limited number of potential locations and usually no more than one cued location. The few studies on multiple locations are equivocal. Pratt (1995) concluded that only the immediately prior attended location remains inhibited (see also Abrams, Oonk, \& Pratt, 1998). Two studies (Danziger, Kingstone, \& Snyder, 1998; Tipper, Weaver, \& Watson, 1996) claim to have shown IOR to three successively cued locations, and Wright (1994; Wright \& Richard, 1996) has demonstrated that IOR can be observed at up to four locations simultaneously. These results are consistent with the notion that IOR can "mark off" examined locations during a serial search procedure. However, Snyder and Kingstone's (2000) data suggest that five or six locations may be the limit, which would reduce the utility of IOR in searching large displays. Although these 
findings suggest that IOR cannot provide the infinite memory required by standard theories, inhibitory tagging of a small number of locations is not incompatible with our data.

None of this is to say that Posner and Cohen's (1984) suggestion is necessarily wrong. A substantial literature exists linking IOR to the oculomotor system (Abrams \& Dobkin, 1994; Kingstone \& Pratt, 1999; Rafal et al., 1989; Sapir, Soroker, Berger, \& Henik, 1999), suggesting that IOR may be a consequence of generating an oculomotor program to saccade to a location, rather than a consequence of attending to a location. On this view, the apparent connection between IOR and covert attention derives from the close coupling of attention and eye movements; although attention can be directed independently of fixation (Posner, 1980), it may be that we cannot make a saccade to a location without first attending to it (Rafal et al., 1989). The time course of IOR is much more compatible with the saccadic system than with a rapid covert attention system. It may be that we can retain the hypothesis that IOR serves to bias the organism toward novelty, as long as we recognize that only eye movements, and not hypothetical shifts of covert attention, are affected. A few studies have examined this question, and the evidence is so far mixed. Largely ignored in the mainstream cognitive literature on visual search have been several studies in the human factors literature that have looked at the question of whether searches that require saccades are "systematic" (memory driven) or "random" (memory free). A number of studies concluded in favor of a random model of search (Bloomfield, 1972; Chan \& Courtney, 1998; Engel, 1977). Arani, Karwan, and Drury (1984) proposed a model of search in which the amount of memory was an explicit parameter. Memory for prior fixations was assumed to degrade as a power function, governed by two free parameters: $\phi$, the (constant) probability of recalling an item on the $i$ th fixation, and $\theta$, the probability of encoding the item in the first place. The probability of recalling on the $i$ th fixation the fact that the area was previously fixated on the $k$ th fixation is then given by

$$
P_{i, k}=\theta \phi^{i-k} \text {. }
$$

If either of the two parameters is set to 0 , the model becomes memory free. If they are both 1 , then the model exhibits perfect memory-driven behavior. Arani et al. (1984) suggested that such a mixed model more accurately reflects actual search behavior, and they proposed that $\theta$ and $\phi$ can be measured directly in eye-movement studies. However, they did not present any data. Similarly, Courtney and Guan (1996) proposed through simulations that a hybrid model may better characterize search behavior.

In a recent study, Klein and MacInnes (1999) monitored eye movements while subjects searched a complex display (taken from the popular "Where's Waldo" books) and found that saccade paths were biased away from a previously fixated locus. Furthermore, probes (black disks) placed at previously fixated locations were more difficult to refixate than were probes at new locations. These results held true only when the search display was maintained throughout probe acquisition, in agreement with the findings of Takeda and Yagi (2000) and Müller and von Mühlenen (2000). Klein and MacInnes used these data to argue that IOR functions as a "foraging facilitator" for the oculomotor system, making the system more likely to fixate new objects than old objects. If this is true, then the virtues of the oculomotor system may compensate for the deficits of the covert attention system. If attention is biased toward the current fixation locus (Carrasco, Evert, Chang, \& Katz, 1995; Carrasco \& Frieder, 1997; Wolfe, O'Neill, \& Bennett, 1998), then moving the eyes every $250-300 \mathrm{msec}$ would serve to prevent attention from resampling the same objects too often. Of course, Klein and MacInnes were able to examine inhibition only at the first and second most recent fixation loci ("one-back" and "two-back"), so we do not know what the capacity of the putative oculomotor inhibitory tagging system might be. Their data are clearly compatible with the mixed models proposed by Arani et al. (1984) and Courtney and Guan (1996), as well as our limited-capacity model.

\section{Visual Marking}

Watson and Humphreys (Olivers, Watson, \& Humphreys, 1999; Watson \& Humphreys, 1997, 1998, 2000) reported a phenomenon they refer to as visual marking. The basic paradigm is as follows: In the "gap" condition, half of the search display is presented at some SOA (typically $1,000 \mathrm{msec}$ ) before the second half. The target is in the second half of the display. In the control condition, all the items are presented simultaneously. Search slopes in the gap condition were half that of the control condition, leading Watson and Humphreys (1997) to conclude that the first half of the display was "marked" as old, so that subjects did not have to search those items when the remainder of the display was presented. These results have been convincingly replicated and extended by Theeuwes, Kramer, and Atchley (1998). At first glance, this would seem to be evidence for a memory process active in aiding search. A serial search system could use such a mechanism to inhibit a group of previously examined objects.

The data presented here argue that neither visual marking nor any other process is operating to keep track of attended locations during search. As with IOR, we have to ask what visual marking $i s$ doing. First, the properties of visual marking suggest that it is not suited for inhibitory tagging in search: Visual marking is capacity demanding (diminished in the presence of a concurrent task) and requires at least $400 \mathrm{msec}$ of exposure to the "old" objects (Watson \& Humphreys, 1997). As with IOR, this time scale would make visual marking a useful inhibitory tagging mechanism only for an attentional processor with a dwell time of 300-500 msec, inconsistent with the dwell time estimates from even our memory-driven models, and near the upper limit of dwell time estimates (Duncan et al., 1994); such dwell times are consistent only with a 
parallel system processing many items at once, in which case there would presumably be no need for an inhibitory tagging system. Second, visual marking has not been shown to be contingent on attending to an object, but rather applies to all those objects whose status is "old." When subjects are first asked to search the old items before the new items are presented, visual marking is not observed (Olivers \& Humphreys, 1999).

\section{Serial Versus Parallel Architectures}

So far, our arguments have been couched in terms of serial processing architectures. However, our findings constrain parallel architectures as well. Consider the unlimited-capacity parallel diffusion model (Ratcliff, Van Zandt, \& McKoon, 1999). Applied to search (Ward \& McClelland, 1989), this takes the form of an array of detectors at each location in the field. In the simplest case, there is one detector at each locus for the target stimulus and one for the distractor stimulus. These detectors gradually accumulate evidence for their specific stimulus at that location. When activation of a target detector exceeds the target threshold, the subject sees a target. When the distractor detector exceeds the distractor threshold, the subject sees a distractor. In an $n$-target search, subjects would wait for $n$ detectors to exceed the "target" threshold before making a "yes" response, and subjects would make a "no" response when all detectors had reached one threshold or the other without enough targets being declared. In such a model, the shape of the critical $m=5$ curve would depend on the distribution of finishing times for the target detectors. If the distribution is normal, for instance, then the diffusion model would make the same (linear) prediction as the memory-driven serial model.

The results of the present experiment constrain the space of search models, both parallel and serial, rather than favoring one general class of architecture over another. However, there is another sense in which these data are relevant to the perennial task of discriminating between parallel and serial theories of attention and vision (Townsend, 1990). Quantitative comparisons between explicit parallel and serial models have been proliferating during the past decade (e.g., Eckstein, 1998; Eckstein, Thomas, Palmer, \& Shimozaki, 2000; Palmer, 1995). Such efforts generally use a standard, memory-driven, serial self-terminating search as the representative of the serial side of the debate. It may be worth revisiting this issue with a memory-free (or limited-memory) serial model pitted against a parallel model.

\section{Adaptive Amnesia?}

Given that search would be unquestionably more efficient if it were memory driven, why has the visual system developed in this seemingly maladaptive way? One answer may be that what we see as failures of memory are in fact side effects of adaptive features of the system (Schacter, 1999). While in the laboratory we may prize perfect recall, the more traces stored in memory, the more difficult it becomes to retrieve a given memory, so the system must be designed to strengthen important memories and weaken unimportant ones (Anderson \& Schooler, 1991). Similarly, although laboratory-based search paradigms may favor memory-driven search, a visual system that invested resources in keeping track of its history may find it difficult to respond to a dynamic environment in which objects (such as predators or prey) were deliberately camouflaged. A memory-free search pattern might also be useful for an organism equipped with an imperfect perceptual system. Object identification can fail at many stages, from the encoding of basic features through to retrieval from long-term storage, so it is likely that many objects will be misclassified. An architecture that discouraged revisiting items might leave the organism vulnerable to early mistakes.

\section{Vision, Attention, and Memory}

In any case, the visual system appears to perform adequately without relying on a rich memory representation. The attentional system acts like a "cognitive miser" (S. E. Taylor, 1981), preferring rapid adequate performance to slow, accurate performance. This is consistent with an emerging body of evidence concerning the transient nature of visual representations. Recent research suggests that, instead of building up a rich, detailed visual representation, the visual system quickly and efficiently constructs a brief sketch of the visual world, which is discarded in the next instant (Irwin, Brown, \& Sun, 1988; Irwin, Zacks, \& Brown, 1990; Levin, Momen, Drivdahl, \& Simons, 2000; Levin \& Simons, 1997; O'Regan, Rensink, \& Clark, 1999; Rayner \& Pollatsek, 1992; Rensink, 2000; Rensink, O'Regan, \& Clark, 1997, 2000; Simons \& Levin, 1998). If vision in general consists of a series of ephemeral and sparse descriptions of the world, then it becomes less surprising that visual search is not accomplished by building up a representation of the attended areas of the scene. A visual system that uses the world as its memory (O'Regan, 1992) would allow the world itself (mediated, perhaps, by a salience map; Koch \& Ullman, 1985) to direct attention, rather than maintaining a map of inhibited locations.

The rationale behind this experiment was that search for multiple, consecutive targets in the same array would give us a better picture of the dynamics of search than a single-target search would. If a memory for the history of attentional deployments existed, subjects should be able to use it to their advantage. Since the data indicate no savings from one target to the next, we conclude that the visual system does not rely on inhibitory tagging to guide attention during search. This conclusion agrees with our previous experiments (Horowitz \& Wolfe, 1998), as well as with the emerging view of "just in time vision." While memory is certainly crucial to perform various components of this search task, it is not employed to improve attentional "foraging."

\section{REFERENCES}

Aвrams, R. A., \& Dobkin, R. S. (1994). Inhibition of return: Effects of attentional cuing on eye movement latencies. Journal of Experimental Psychology: Human Perception \& Performance, 20, 467-477. 
Abrams, R. A., Oonk, H. M., \& Pratt, J. (1998). Fixation point offsets facilitate endogenous saccades. Perception \& Psychophysics, 60, 201-208.

Anderson, J. R., \& Schooler, L. J. (1991). Reflections of the environment in memory. Psychological Science, 2, 396-408.

Arani, T., Karwan, M. H., \& Drury, C. G. (1984). A variable-memory model of search. Human Factors, 26, 631-639.

Barbur, J. L., Forsyth, P. M., \& Wooding, D. S. (1993). Eye movements and search performance. In D. Brogan, A. Gale, \& K. Carr (Eds.), Visual search 2 (pp. 253-264). London: Taylor \& Francis.

Bloomfield, J. R. (1972). Visual search in complex fields: Size differences between target disc and surrounding discs. Human Factors, 14, 139-148.

Briand, K. A., \& Klein, R. M. (1987). Is Posner's "beam" the same as Treisman's "glue"? On the relation between visual orienting and feature integration theory. Journal of Experimental Psychology: Human Perception \& Performance, 13, 228-241.

Carrasco, M., Evert, D. L., Chang, I., \& Katz, S. M. (1995). The eccentricity effect: Target eccentricity affects performance on conjunction searches. Perception \& Psychophysics, 57, 1241-1261.

Carrasco, M., \& Frieder, K. S. (1997). Cortical magnification neutralizes the eccentricity effect in visual search. Vision Research, 37, 63-82.

Chan, A. H., \& Courtney, A. J. (1998). Revising and validating the random search model for competitive search. Perceptual \& Motor Skills, 87, 251-260.

Cheal, M., Chastain, G., \& Lyon, D. R. (1998). Inhibition of return in visual identification tasks. Visual Cognition, 5, 365-388.

CHUn, M. M., \& JIANG, Y. (1998). Contextual cueing: Implicit learning and memory of visual context guides spatial attention. Cognitive Psychology, 36, 28-71.

Chun, M. M., \& Potter, M. C. (1995). A two-stage model for multiple target detection in rapid serial visual presentation. Journal of Experimental Psychology: Human Perception \& Performance, 21, 109 127.

Chun, M. M., \& Wolfe, J. M. (1996). Just say no: How are visual searches terminated when there is no target present? Cognitive Psychology, 30, 39-78.

Courtney, A. J., \& Guan, L. (1996). Visual search performance with complete and incomplete visual lobe areas. Ergonomics, 39, 749-756.

Danziger, S., Kingstone, A., \& SNyder, J. J. (1998). Inhibition of return to successively stimulated locations in a sequential visual search paradigm. Journal of Experimental Psychology: Human Perception \& Performance, 24, 1467-1475.

DownING, P. E. (1999). Attentional consequences of maintaining an object in visual working memory. Investigative Ophthalmology \& Visual Science, 40, S753.

Duncan, J., \& Humphreys, G. (1989). Visual search and stimulus similarity. Psychological Review, 96, 433-458.

Duncan, J., \& Humphreys, G. (1992). Beyond the search surface: Visual search and attentional engagement. Journal of Experimental Psychology: Human Perception \& Performance, 18, 578-588.

Duncan, J., WARD, R., \& Shapiro, K. L. (1994). Direct measurement of attentional dwell time in human vision. Nature, 369, 313-315.

EcKSTEIN, M. P. (1998). The lower visual search efficiency for conjunctions is due to noise and not serial attentional processing. Psychological Science, 9, 111-118.

Eckstein, M. P., Thomas, J. P., Palmer, J., \& Shimozaki, S. S. (2000). A signal detection model predicts the effects of set size on visual search accuracy for feature, conjunction, triple conjunction, and disjunction displays. Perception \& Psychophysics, 62, 425-451.

ENGEL, F. L. (1977). Visual conspicuity, visual search and fixation tendencies of the eye. Vision Research, 17, 95-108.

ERIKSEN, C. W. (1988). A source of error in attempts to distinguish coactivation from separate activation in the perception of redundant targets. Perception \& Psychophysics, 44, 191-193.

Estes, W. K., \& TAYloR, H. A. (1966). Visual detection in relation to display size and redundancy of critical elements. Perception \& Psychophysics, 1, 9-16.

Gibson, B. S., Li, L., Skow, E., Brown, K., \& Cooke, L. (2000).
Searching for one versus two identical targets: When visual search has a memory. Psychological Science, 11, 324-327.

Grossberg, S., Mingolla, E., \& Ross, W. D. (1994). A neural theory of attentive visual search: Interactions of boundary, surface, spatial, and object representations. Psychological Review, 101, 470-489.

Hillstrom, A. P. (2000). Repetition effects in visual search. Perception \& Psychophysics, 62, 800-817.

Hillstrom, A. P., \& Logan, G. D. (1998). Decomposing visual search: Evidence of multiple item-specific skills. Journal of Experimental Psychology: Human Perception \& Performance, 24, 1385-1398.

Hock, H. S., Rosenthal, A., \& Stenquist, P. (1985). The category effect in visual search: Practice effects on catch trials. Perception \& Psychophysics, 37, 73-80.

Horowitz, T. S., \& Wolfe, J. M. (1998). Visual search has no memory. Nature, 394, 575-577.

IRwIN, D. E., BRown, J. S., \& Sun, J.-S. (1988). Visual masking and visual integration across saccadic eye movements. Journal of Experimental Psychology: General, 117, 276-287.

IRwiN, D. E., ZACKS, J. L., \& Brown, J. S. (1990). Visual memory and the perception of a stable visual environment. Perception \& Psychophysics, 47, 35-46.

Johnson, N. L., \& Kotz, S. (1977). Urn models and their applications. New York: Wiley.

Kingstone, A., \& Pratt, J. (1999). Inhibition of return is composed of attentional and oculomotor processes. Perception \& Psychophysics, 61, 1046-1054.

KLEIN, R. (1988). Inhibitory tagging system facilitates visual search. Nature, 334, 430-431.

KLeIn, R. M., \& MacInnes, W. J. (1999). Inhibition of return is a foraging facilitator in visual search. Psychological Science, 10, 346-352.

Klein, R. M., \& TAYloR, T. L. (1994). Categories of cognitive inhibition with reference to attention. In D. Dagenbach \& T. H. Carr (Eds.), Inhibitory processes in attention, memory, and language (pp. 113150). San Diego: Academic Press.

Косн, C., \& Ullman, S. (1985). Shifts in selective visual attention: Towards the underlying neural circuitry. Human Neurobiology, 4, 219 227.

KRUEGER, L. E. (1984). The category effect in visual search depends on physical rather than conceptual differences. Perception \& Psychophysics, 35, 558-564.

Levin, D. T., Momen, N., Drivdahl, S. B., \& Simons, D. J. (2000). Change blindness blindness: The metacognitive error of overestimating change-detection ability. Visual Cognition, 7, 397-412.

Levin, D. T., \& Simons, D. J. (1997). Failure to detect changes to attended objects in motion pictures. Psychonomic Bulletin \& Review, 4, 501-506.

Lupiáñez, J., Milán, E. G., Tornay, F. J., Madrid, E., \& Tudela, P. (1997). Does IOR occur in discrimination tasks? Yes, it does, but later. Perception \& Psychophysics, 59, 1241-1254.

LupiáñeZ, J., \& Milliken, B. (1999). Inhibition of return and the attentional set for integrating versus differentiating information. Journal of General Psychology, 126, 392-418.

Maljkovic, V., \& Nakayama, K. (1994). Priming of pop-out: I. Role of features. Memory \& Cognition, 22, 657-672.

Maljkovic, V., \& Nakayama, K. (1996). Priming of pop-out: II. The role of position. Perception \& Psychophysics, 58, 977-991.

MAY LOR, E. A (1985). Facilitatory and inhibitory components of orienting in visual space. In M. I. Posner \& O. S. M. Marin (Eds.), Attention and performance XI (pp. 189-204). Hillsdale, NJ: Erlbaum.

Miller, J., \& Lopes, A. (1991). Bias produced by fast guessing in distribution-based tests of race models. Perception \& Psychophysics, 50, 584-590.

Mondor, T. A., Breau, L. M., \& Milliken, B. (1998). Inhibitory processes in auditory selective attention: Evidence of location-based and frequency-based inhibition of return. Perception \& Psychophysics, 60, 296-302.

Moore, C. M., \& Wolfe, J. M. (in press). Getting beyond the serial/parallel debate in visual search: A hybrid approach. In K. Shapiro (Ed.), The limits of attention: Temporal constraints on human information processing. Oxford: Oxford University Press. 
Moray, N. (1969). Attention: Selective processing in vision and hearing. London: Hutchinson.

Moyer, R. S., \& Landauer, T. K. (1967). Time required for judgements of numerical inequality. Nature, 215, 1519-1520.

Müller, H., \& VON MüHLEnEN, A. (2000). Probing distractor inhibition in visual search: Inhibition of return. Journal of Experimental Psychology: Human Perception \& Performance, 26, 1591-1605.

Olivers, C. N. L., \& Humphreys, G. W. (1999, November). Visual marking is distinct from inhibition of return. Paper presented at the annual meeting of the Psychonomic Society, Los Angeles.

Olivers, C. N. L., Watson, D. G., \& Humphreys, G. W. (1999). Visual marking of locations and feature maps: Evidence from withindimension defined conjunctions. Quarterly Journal of Experimental Psychology, 52A, 679-715.

Oonk, H. M., \& ABrams, R. A. (1998). New perceptual objects that capture attention produce inhibition of return. Psychonomic Bulletin \& Review, 5, 510-515.

O'Regan, J. K. (1992). Solving the "real" mysteries of visual perception: The world as an outside memory. Canadian Journal of Psychology, 46, 461-488.

O'Regan, J. K., Rensink, R. A., \& Clark, J. J. (1999). Change-blindness as a result of "mudsplashes." Nature, 398, 34.

Palmer, J. (1995). Attention in visual search: Distinguishing four causes of a set-size effect. Current Directions in Psychological Science, 4, 118-123.

Posner, M. I. (1980). Orienting of attention: The VIIth Sir Frederic Bartlett Lecture. Quarterly Journal of Experimental Psychology, 32, 3-25.

Posner, M. I., \& Cohen, Y. (1984). Components of attention. In H. Bouma \& D. G. Bouwhuis (Eds.), Attention and performance $X$ (pp. 55-66). Hillsdale, NJ: Erlbaum.

Pratt, J. (1995). Inhibition of return in a discrimination task. Psychonomic Bulletin \& Review, 2, 117-120.

Pratt, J., \& Abrams, R. A. (1999). Inhibition of return in discrimination tasks. Journal of Experimental Psychology: Human Perception \& Performance, 25, 229-242.

Pratt, J., Kingstone, A., \& Khoe, W. (1997). Inhibition of return in location- and identity-based choice decision tasks. Perception \& Psychophysics, 59, 964-971.

Prinzmetal, W., Presti, D. E., \& Posner, M. I. (1986). Does attention affect visual feature integration? Journal of Experimental Psychology: Human Perception \& Performance, 12, 361-369.

Pylyshyn, Z (1989). The role of location indexes in spatial perception: A sketch of the FINST spatial-index model. Cognition, 32, 65-97.

Rafal, R. D., Calabresi, P. A., Brennan, C. W., \& Sciolto, T. K. (1989). Saccade preparation inhibits reorienting to recently attended locations. Journal of Experimental Psychology: Human Perception \& Performance, 15, 673-685.

Ratcliff, R., Van Zandt, T., \& McKoon, G. (1999). Connectionist and diffusion models of reaction time. Psychological Review, 106, 261-300.

Rayner, K., \& Pollatsek, A. (1992). Eye movements and scene perception. Canadian Journal of Psychology, 46, 342-376.

Rensink, R. A. (2000). Seeing, sensing, and scrutinizing. Vision Research, 40, 1469-1487.

Rensink, R. A., O' Regan, J. K., \& Clark, J. J. (1997). To see or not to see: The need for attention to perceive changes in scenes. Psychological Science, 8, 368-373.

Rensink, R. A., O'Regan, J. K., \& Clark, J. J. (2000). On the failure to detect changes in scenes across brief interruptions. Visual Cognition, 7, 127-145.

Reuter-Lorenz, P. A., JhA, A. P., \& Rosenquist, J. N. (1996). What is inhibited in inhibition of return? Journal of Experimental Psychology: Human Perception \& Performance, 22, 1241-1254.

SAPIr, A., Soroker, N., Berger, A., \& Henik, A. (1999). Inhibition of return in spatial attention: Direct evidence for collicular generation. Nature Neuroscience, 2, 1053-1054.

SCHACTER, D. L. (1999). The seven sins of memory: Insights from psychology and cognitive neuroscience. American Psychologist, 54, 182-203.

SCHNEIDER, W., \& SHIFFrin, R. M. (1977). Controlled and automatic human information processing: I. Detection, search, and attention. Psychological Review, 84, 1-66.

SCHNEIDER, W. X. (1995). VAM: A neuro-cognitive model for visual attention control of segmentation, object recognition, and space-based motor action. Visual Cognition, 2, 331-376.

Simons, D. J., \& LeVIn, D. T. (1998). Failure to detect changes to people during a real-world interaction. Psychonomic Bulletin \& Review, 5, 644-649.

SNyder, J. J., \& Kingstone, A. (2000). Inhibition of return and visual search: How many separate loci are inhibited? Perception \& Psychophysics, 62, 452-458.

Sternberg, S. (1969). High-speed scanning in human memory. Science, 153, 652-654.

TAKEDA, Y., \& YAGI, A. (2000). Inhibitory tagging in visual search can be found if search stimuli remain visible. Perception \& Psychophysics, 62, 927-934.

TAYLOR, S. E. (1981). A categorization approach to stereotyping. In D. L. Hamilton (Ed.), Cognitive processes in stereotyping and intergroup behavior (pp. 88-114). Hillsdale, NJ: Erlbaum.

TAY LOR, T. L., \& KLEIN, R. M. (1998). On the causes and effects of inhibition of return. Psychonomic Bulletin \& Review, 5, 625-643.

Terry, K. M., VAldes, L. A., \& Neill, W. T. (1994). Does "inhibition of return" occur in discrimination tasks? Perception \& Psychophysics, 55, 279-286.

Theeuwes, J., Kramer, A. F., \& Atchley, P. (1998). Visual marking of old objects. Psychonomic Bulletin \& Review, 5, 130-134.

Tipper, S. P., Driver, J., \& Weaver, B. (1991). Object-centered inhibition of return of visual attention. Quarterly Journal of Experimental Psychology: Human Experimental Psychology, 43A, 289-298.

Tipper, S. P., Weaver, B., Jerreat, L. M., \& Burak, A. L. (1994). Object-based and environment-based inhibition of return of visual attention. Journal of Experimental Psychology: Human Perception \& Performance, 20, 478-499.

Tipper, S. P., Weaver, B., \& Watson, F. L. (1996). Inhibition of return to successively cued spatial locations: Commentary on Pratt and Abrams (1995). Journal of Experimental Psychology: Human Perception \& Performance, 22, 1289-1293.

Townsend, J. T. (1990). Serial vs. parallel processing: Sometimes they look like Tweedledum and Tweedledee but they can (and should) be distinguished. Psychological Science, 1, 46-54.

Treisman, A. (1985). Preattentive processing in vision. Computer Vision, Graphics, \& Image Processing, 31, 156-177.

Treisman, A., \& Gelade, G. (1980). A feature-integration theory of attention. Cognitive Psychology, 12, 97-136.

Treisman, A., \& Sato, S. (1990). Conjunction search revisited. Journal of Experimental Psychology: Human Perception \& Performance, 16, 459-478.

WARD, R. \& McClelland, J. L. (1989). Conjunctive search for one and two identical targets. Journal of Experimental Psychology: Human Perception \& Performance, 15, 664-672.

Watson, D. G., \& Humphreys, G. W. (1997). Visual marking: Prioritizing selection for new objects by top-down attentional inhibition of old objects. Psychological Review, 104, 90-122.

Watson, D. G., \& Humphreys, G. W. (1998). Visual marking of moving objects: A role for top-down feature-based inhibition in selection. Journal of Experimental Psychology: Human Perception \& Performance, 24, 946-962.

Watson, D. G., \& Humphreys, G. W. (2000). Visual marking: Evidence for inhibition using a probe-dot detection paradigm. Perception \& Psychophysics, 62, 471-481.

Wolfe, J. M. (1994). Guided Search 2.0: A revised model of visual search. Psychonomic Bulletin \& Review, 1, 202-238.

Wolfe, J. M., O'Neill, P., \& Bennett, S. C. (1998). Why are there eccentricity effects in visual search? Visual and attentional hypotheses. Perception \& Psychophysics, 60, 140-156.

Wolfe, J. M., \& PoKorny, C. W. (1990). Inhibitory tagging in visual search: A failure to replicate. Perception \& Psychophysics, 48, $357-$ 362.

Wolford, G. L., Wessel, D. L., \& Estes, W. K. (1968). Further evidence concerning scanning and sampling assumptions of visual detection models. Perception \& Psychophysics, 3, 439-444. 
Wright, R. D. (1994). Shifts of visual attention to multiple simultaneous location cues. Canadian Journal of Experimental Psychology, 48, 205-217.

Wright, R. D., \& Richard, C. M. (1996). Inhibition of return at multiple locations in visual space. Canadian Journal of Experimental Psychology, 50, 324-327.

Yantis, S., Meyer, D. E., \& Smith, J. E. K. (1991). Analyses of multinomial mixture distributions: New test for stochastic models of cognition and action. Psychological Bulletin, 110, 350-374.

ZENGER, B., \& FAHLE, M. (1997). Missed targets are more frequent than false alarms: A model for error rates in visual search. Journal of Experimental Psychology: Human Perception \& Performance, 23, 1783-1791.

\section{NOTES}

1. At this point, we are discussing only serial models of attention and search. However, the competing class of parallel models is built on the same assumption. We explore this issue in more depth in the General Discussion.
2. For $m>2$, the main effect of $n$ was always significant, and the $n$ $\times m$ interaction was significant for $m>3(p=.09$ for $m=2)$. However, we believe that these effects are better understood in the context of our analysis of trend, rather than in the traditional ANOVA.

3. This is an oversimplification. Subjects can get away with less than exhaustive searches when $n$ and $m$ are far apart (see the section on errors). The overall point still holds, however.

4. We are grateful to Art Kramer for this suggestion.

5. Inconveniently, the method for computing the probability of finding exactly $m^{\prime}$ targets in a sample of $C$ items is valid only for $C<$ (set size $-m$ ) (see Johnson \& Kotz, 1977, p. 80).

6. Adding this search through memory procedure to the memory-free model will substantially improve the fit to the data. However, since we were primarily interested in comparisons with the memory-driven model (which needs no such component), we thought it would be unfair to add an extra parameter to the memory-free model.

(Manuscript received September 14, 1999; revision accepted for publication May 22, 2000.) 